\title{
Todo lo que nunca pensaste que los alumnos sub 18 sabían sobre proyectos
}

\section{Everything You Never Thought Under 18 Students Knew about Projects}

Isabel Ramos, Javier J. Gutiérrez, Carlos Arévalo, Francisco J. Domínguez, Juan M. Cordero, Manolo Mejías

Departamento de Lenguajes y Sistemas Informáticos, Escuela Técnica Superior de Ingeniería Informática, Universidad de Sevilla. España\{iramos, javierj, carlosarevalo, fjdominguez, cordero, risoto\}@us.es

\section{Resumen}

Organizar excursiones o meriendas, montar un equipo del juego on-line de moda, estudiar en grupo y muchas cosas más son actividades habituales en jóvenes (y no tan jóvenes). Las actividades anteriores pueden enmarcarse en la definición de proyecto, ya que todas cuentan con un marco temporal definido, tienen recursos limitados que es necesario gestionar y plantean objetivos que se desea cumplir. Sin embargo, basándonos en nuestra experiencia docente en asignaturas universitarias relacionadas con la gestión y dirección de proyectos informáticos, la enseñanza de esta materia es difícil. Al ser asignaturas de últimos cursos universitarios los alumnos llegan con malos hábitos adquiridos. El objetivo de este trabajo es doble: 1) Exponer cómo en actividades cotidianas aplicamos conocimientos de gestión de proyectos, aunque muchas veces no somos conscientes de ello. Queremos mostrar a nuestros futuros estudiantes el uso de buenas prácticas de gestión de proyectos tomando como referencia la Guía de los Fundamentos para la Dirección de Proyectos (Guía del PMBOK®) del Project Management Institute (PMI) y aplicándolo a la organización de una barbacoa. Así, el alumnado aprenderá que detrás de cada una de las preguntas que se plantean: ¿dónde lo haremos?, ¿cuántos seremos?, ¿qué llevaremos?, ¿cuándo? y otras tantas preguntas, se esconden buenas prácticas de gestión de proyectos; y 2) Plantear al profesorado, tanto universitario como no universitario, la importancia de ir incorporando en etapas tempranas de la formación de nuestro alumnado conceptos básicos sobre diferentes disciplinas aplicadas de forma amena y motivadora. Nuestros jóvenes saben más de gestión de proyectos de lo que ellos mismos creen y ayudarles a sacar este conocimiento a la luz les ayudará a gestionar un proyecto de forma natural cuando lleguen a la universidad. Para ello expondremos técnicas y herramientas sencillas para que su barbacoa sea un éxito.

\section{Palabras Clave}

Gestión y dirección de proyectos; Planificación; Procesos gestión de recursos; Riesgos; Alcance; Interesados

\section{Abstract}

Organizing excursions or afternoon snacks, putting together a team of the most fashionable online game as well as studying in groups and many other things are common activities among young people. Such activities are framed in a project, since they all have a defined time framework, they have limited resources that need to be managed and they set goals that must be met. However, according to our professional experience in university subjects related to IT projects management, teaching this module is difficult because it is taught in the last courses of their degrees and students may reach this level with bad acquired habits. For this reason, we have set out two objectives to carry out this work: On the one hand, to identify everyday activities where we usually apply project management skills without being aware of it. We aim to show our future students the use of good project management practices, following the Project Management Fundamentals Guide (PMBOK@ Guide) of the Project Management Institute (PMI), and how to apply them. In this case, we have taken the organization of a barbecue as an example. Thus, the new university student will learn that questions such as where and when will it take place? how many people will be there? what will we bring? among others, hide good project management practices. Young people know more about project management than they think they know. Therefore, bringing this knowledge to light will help them manage a project, naturally and unconsciously, when they reach university degrees. For this purpose, we will display simple techniques and tools in order to make a barbecue be a successful event. On the other hand, to make teachers, both university and nonuniversity, aware of the importance of incorporating basic concepts related to project management, in an entertaining and motivating way, in the early stages of students' education.

\section{Keywords}

Management and direction of projects; Planning, Processes resources management; Risks; Scope; Stakeholders 


\section{Introducción}

Basándonos en nuestra experiencia, la enseñanza de gestión y dirección de proyectos no es siempre fácil, tanto para alumnos preuniversitarios como para los alumnos universitarios. Mucha de la literatura existente es aburrida y poco práctica. Los ejemplos suelen estar alejados de la realidad y las inquietudes del alumnado.

Organizar excursiones, viajes, meriendas, cumpleaños, montar un equipo del juego on-line, estudiar en grupo, y muchas cosas más son actividades habituales en los jóvenes de menos de 18 años que pueden enmarcarse dentro de la definición de proyecto, ya que todas cuentan con un marco temporal definido, todas tienen recursos limitados y todas plantean objetivos que se desea cumplir en un contexto de incertidumbre. Por tanto, coinciden con la definición de proyecto que utilizamos en nuestras clases y que se desarrollará en el siguiente apartado.

Este artículo tiene como objetivo presentar la experiencia de los autores en gestión y dirección de proyectos para facilitar la enseñanza de esta materia de manera que sea más accesible y amena para el alumnado. Para ello, se muestra cómo muchos de los procesos y áreas de conocimiento de gestión de proyectos están presentes en muchas actividades que organizan nuestros jóvenes sin que ellos sean conscientes. Proponemos irnos de barbacoa, pero: ¿en qué fecha?, ¿dónde iremos?, ¿quiénes vendrán?, ¿costará mucho?, ¿nos dejarán nuestros padres?, etc. Buscamos contestar a cada una de estas y otras preguntas que irán surgiendo durante la organización de la barbacoa, siguiendo el estándar internacional del PMI® (www.pmi.org), la Guía de los fundamentos para la Dirección de Proyectos (Guía del PMBOK®) (Project Management Institute [PMI], 2014), en adelante PMBOK, para que podamos ir a la barbacoa, disfrutarla y volver contentos para empezar a pensar en la siguiente. Aunque esta vez y las siguientes, con el conocimiento adquirido la organización será mucho más rápida y las posibilidades de éxito mucho mayor. Eso es lo que llamamos, en dirección y gestión de proyectos, analizar las lecciones aprendidas de un proyecto que acaba de finalizar al objeto de mejorar nuestras estimaciones para futuros proyectos.

Finalmente nos gustaría utilizar este trabajo para plasmar la importancia que tiene, en los primeros años de formación, el ir introduciendo conocimientos y habilidades que en edades más avanzadas tendrán que aplicarse a problemas más complejos. Propuestas como el proyecto TACCLE3 (GarcíaPeñalvo, 2016) debería ser extensiva en otros ámbitos del conocimiento.

Este trabajo está estructurado según lo siguiente: en el apartado 2, comenzamos con la definición del Proyecto Barbacoa y sus aspectos más importantes alternándolos con los Procesos y Áreas de Conocimiento que utilizaremos según el PMBOK (PMI, 2014), en el apartado 3 aplicamos todo lo 
anterior para organizar el Proyecto Barbacoa, en el apartado 4 proponemos algunas herramientas sencillas para facilitar la realización de un plan de proyectos, su control y ejecución; y algunas lecturas relacionadas que pueden aportar información de interés para motivar al alumnado en los temas tratados en este trabajo, el apartado 5 está dedicado a recoger las conclusiones de este trabajo, finalmente aportamos los agradecimientos y la bibliografía referenciada.

\section{El proyecto Barbacoa y el PMBOK}

En este apartado iremos simultaneando las decisiones que tomará el alumnado para celebrar la barbacoa con algunos de los procesos y áreas de conocimiento del PMBOK. Comprobaremos que, aunque nuestro alumnado no llegue al nivel de formalidad del PMBOK porque lo desconocen, sí lo sigue en gran medida.

\subsection{Descripción de la barbacoa: ¿Es un proyecto?}

Seguramente nuestro alumnado de forma escrita u oral hará una descripción de lo que quiere hacer muy parecida a lo siguiente, en la que ya está implícita la justificación y los objetivos de lo que se pretende hacer:

Los alumnos del Grupo 3 de cuarto curso de ESO decidimos realizar una barbacoa para celebrar el final de los exámenes. Muchos de nosotros no nos veremos hasta después del verano y nos gustaría hacer una fiesta antes de marcharnos.

Existen otros grupos que suelen organizar actividades de este tipo cuando se termina el curso y a nosotros nos gustaría también hacer algo parecido.

Nuestra idea es celebrar una barbacoa al aire libre en el jardín de la casa de un compañero (José Manuel Pérez) a la que asistamos toda la clase y solo la clase. Es un sitio que está bien comunicado y ya en otras ocasiones hemos celebrado allí algunas quedadas. En el jardín hay una barbacoa muy bien montada y los padres de José Manuel son muy simpáticos

\footnotetext{
El equipo organizador se encargará de las compras necesarias (comida, bebidas, servilletas, vasos, platos, cubiertos, etc.). Como muy tarde tendremos que empezar con los preparativos el día 5 de junio para que el día 24 por la mañana (día de la barbacoa) esté todo listo. El día 25 lo dedicaremos a recogerlo todo y dejar el jardín limpio, hacer recuento final y repartirnos lo que haya sobrado. Hemos acordado con los padres de José y con los vecinos colindantes que la barbacoa empezará a las 14h y que acabará como muy tarde a las $01 \mathrm{~h}$ del día 25, que solamente asistiremos los que somos de la clase y que el espacio para realizar la barbacoa será solo el jardín.
} 
Hemos hablado con unos amigos de otros grupos que celebran fiestas parecidas y estiman que, si lo hacemos en casa de algún amigo que ya tenga barbacoa instalada en el jardín, nos podríamos gastar unos 400 euros.

Nuestro objetivo con la barbacoa es conocernos mejor y, sobre todo, pasarlo bien.

La persona que se encargará de todo será nuestra delegada de clase (Rosa Martínez) que junto con los que se han ofrecido (José Manuel Pérez, Mario Ramírez, Juana Muñiz, Alejandro Silva y Nuria Fernández) formarán el equipo de trabajo.

Mario Ramírez, se ha ofrecido a ser el encargado de recoger el dinero y administrarlo para hacer todas las compras y gastos previstos. También se encargará de pedir más dinero si hubiese que aumentar la cuota que hemos calculado.

Tenemos que decidir qué grupos de WhatsApp se crearán, quién los gestionará y qué información se enviará.

Ya sabemos, mucho, de lo que quiere hacer el alumnado, ahora lo primero que tenemos que preguntarnos es: ¿es esto un proyecto?

Cualquier definición de proyecto incluye que:

- Está formado por un conjunto de actividades interrelacionadas entre sí llevadas a cabo por un equipo para cumplir uno o más objetivos.

- Se transforman un conjunto de recursos en unos resultados con un determinado nivel de calidad.

- Tiene una duración (fecha de inicio y finalización) y presupuesto estimados.

- Conlleva un determinado nivel de incertidumbre que dependerá en gran medida (aunque no siempre) de los requisitos del mismo. Todo esto hace, frente a las operaciones repetitivas, que cada proyecto sea único e irrepetible.

Si repasamos la descripción que ha dado el alumnado sobre lo que quiere hacer y la definición de proyecto que hemos aportado, podemos afirmar que organizar una barbacoa sí es un proyecto: la organización y ejecución de la barbacoa conlleva la realización de un conjunto de actividades que consumen recursos (horas dedicadas del equipo, un espacio, víveres y otros suministros), para cubrir unos objetivos (fomentar el compañerismo, conocerse y pasarlo bien), llevadas a cabo por un equipo (Rosa, José Manuel, Mario, Juana, Alejandro y Silvia), es decir, requiere esfuerzo que se realizará durante un tiempo, con una fecha de inicio y de finalización (del 5 al 25 de junio), que costará dinero (400 euros) y tendrán que elaborar un presupuesto, que esperan que cumplan con unas expectativas 
o nivel de calidad (ambiente agradable, buena compañía, buena comida, etc.) y que aunque pondrán su mayor esfuerzo para que todo salga bien es posible que algo falle (mal tiempo, la negativa de los vecinos, conflictos personales, etc.). También esta barbacoa será única e irrepetible. Habrá otras, pero las expectativas, los objetivos, los asistentes, el lugar, y otros aspectos serán diferentes. Por tanto, a partir de ahora a este proyecto le llamaremos Proyecto Barbacoa o simplemente Proyecto. Para este Proyecto la clase completa será simultáneamente financiadores (sponsor) y usuarios. El equipo será el equivalente a la parte contratada para dirigirlo y gestionarlo.

Formalmente y en un primer estadio, los aspectos más significativos de un proyecto se recogen en el Acta de Constitución (apartado 3.1, (PMI, 2014)). Una vez aprobado el acta por el sponsor, la persona responsable del proyecto puede comenzar con la definición del Plan para la Dirección del Proyecto en el que se definirán con mayor nivel los requisitos técnicos, de alcance, tiempo, coste y calidad de un proyecto (PMI, 2014).

En el caso que nos ocupa, con una buena y completa redacción del Acta de Constitución del Proyecto Barbacoa sería suficiente. La realización del Plan completo nos daría para otro trabajo.

\section{Procesos y áreas de conocimiento del PMBOK aplicados al Proyecto Barbacoa}

\subsection{Grupos de procesos principales}

La Guía de los Fundamentos para la Dirección de Proyectos (Guía del PMBOK®) (PMI, 2014) es un conjunto de buenas prácticas para llevar a cabo de manera sistemática la dirección y gestión de un proyecto. En esta sección destacaremos solamente los aspectos más importantes para abordar el Proyecto Barbacoa.

En la Figura 1, se representan los cinco grupos principales de procesos que se deben llevar a cabo para la realización de un proyecto y las dependencias establecidas entre los mismos. La salida de un proceso normalmente se convierte en la entrada de otro proceso, o es un entregable del proyecto.

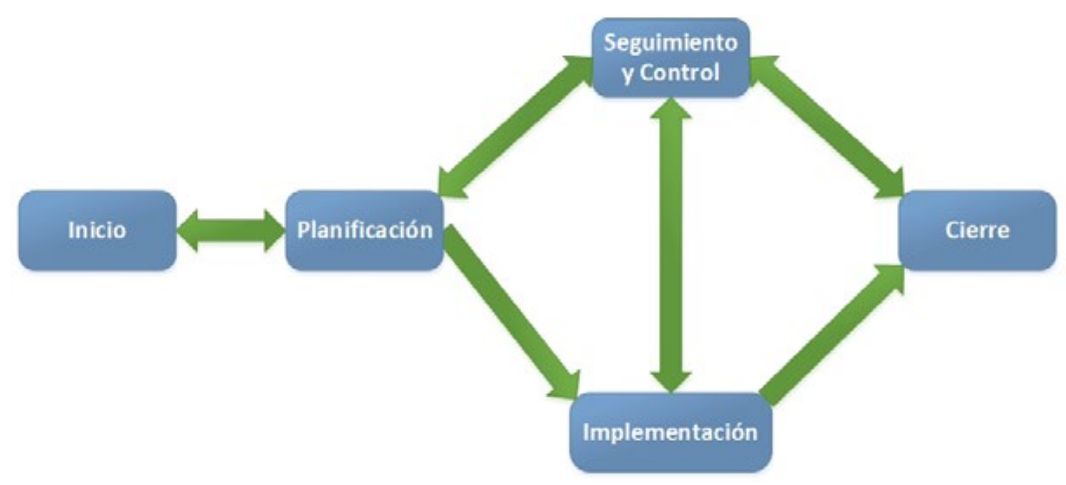


- Procesos de Inicio: grupo de procesos donde se definen las características del proyecto, a muy alto nivel, recogidas en el Acta de Constitución, firmada por el sponsor y en la que se nombra a la persona responsable del proyecto y se le autoriza a comenzar con la planificación del mismo.

- Procesos de Planificación: grupo de procesos que definen con detalle la planificación del proyecto para establecer las líneas de base (del coste, tiempo y alcance) contra las cuales se gestionará la implementación del mismo. Los cambios importantes que ocurren a lo largo del ciclo de vida del proyecto generan, normalmente, la necesidad de reconsiderar uno o más de lo procesos de planificación y, posiblemente, alguno de los procesos de inicio siempre con el visto bueno del sponsor.

- Procesos de Ejecución o Implementación: procesos que llevan a cabo las actividades de gestión y dirección del proyecto y de apoyo en la producción de los entregables de acuerdo con lo planificado para realizar y completar el trabajo definido en el plan para la dirección del proyecto. Estos procesos implican una importante coordinación de las personas y de los recursos, así como integrar y realizar las actividades del proyecto acorde con el plan establecido.

- Procesos de Seguimiento y Control: procesos que miden y controlan el avance del proyecto según el plan del mismo al objeto de tomar acciones preventivas y correctivas, si fuese necesario, y proponer los cambios oportunos para lograr los objetivos establecidos. Podemos decir, que estos procesos "vigilan" el trabajo realizado durante la ejecución del proyecto para ajustarnos en cada momento al plan establecido.

- Procesos de Cierre: Una vez finalizado el proyecto, estos procesos permiten cerrar formalmente el proyecto, tanto desde el punto de vista del cliente como de la entidad que lo ejecuta y las relaciones establecidas con los proveedores. Finalmente se redactan las lecciones aprendidas durante la ejecución del proyecto para "aprender" de la experiencia adquirida.

Estos grupos principales de procesos se descomponen en otros procesos más específicos (subprocesos) y se clasifican, según el PMBOK, dentro de diez áreas de conocimiento, cada área de conocimiento constituye un conjunto específico de conocimientos para poder abordar determinados procesos.

¿Realiza el alumnado el Proyecto Barbacoa según la estructura de la Figura 1? En primer lugar, comienzan con los Procesos de Inicio poniéndose de acuerdo en los aspectos más importantes del proyecto, nombrando a la persona que lo liderará (Rosa) y viendo quiénes serán los interesados en el mismo, a partir de este momento Rosa y el equipo comenzarán a realizar el plan que van a seguir, Planificación (recopilar requisitos, definir las actividades a realizar, estimar la duración de cada una, hacer un cronograma, calcular el presupuesto, definir la calidad, definir las comunicaciones, identificar 
los riesgos, etc.) para determinar, con detalle, el tamaño, el tiempo de duración, el presupuesto, la calidad deseada y cómo se van a comunicar tanto los miembros del equipo organizador como con el resto de interesados. Una vez definido el plan comenzaremos con la gestión del mismo (compras, hablar con los vecinos, recoger el dinero, etc.) y lo llevarán a cabo en la Ejecución de la barbacoa. Durante la barbacoa estarán pendientes de que no falte nada y que todo vaya según lo previsto, Seguimiento y Control, tanto en el alcance, la calidad y otros aspectos como el tiempo de duración que hemos acordado y el presupuesto estimado. Si vemos que algo se está saliendo del plan tendremos que realizar algunos cambios, siempre con el visto bueno de Rosa. Finalmente, cuando llegue la hora establecida tendremos que finalizar el Proyecto, Cierre, recogiendo el jardín, analizando cómo ha ido todo y seguramente dándoles las gracias a los padres de José Manuel. Si todo ha salido como desean seguro que volverán a quedar para organizar otra barbacoa, posiblemente con la experiencia adquirida y sabiendo lo bien que ha funcionado el equipo, en lugar de hacerla en casa de un amigo la harán en el campo e invitarán a más compañeros. Es decir, aunque hagan otra barbacoa será otra diferente (otro proyecto) pero la organizaran mejor y con más rapidez por lo que han aprendido.

\subsection{Subprocesos más significativos}

En la Tabla 1, se muestran los subprocesos, que hemos considerado más significativos para el propósito que nos ocupa, respetando la denominación de los subprocesos recogidos en (PMI, 2014) y algunas de las preguntas, no todas, que hemos aportado en un lenguaje coloquial (próximo a nuestro alumnado) para mostrar lo que se pretende abordar en cada subproceso y a las que tendrán que responder. Estos subprocesos, de manera consciente o no, lo llevará a cabo el alumnado para el Proyecto Barbacoa. Se ha valorado el poder introducir notaciones gráficas tanto para definir las actividades y tareas de un proyecto como para la propia organización del proyecto, con el fin de transmitir de una manera más sencilla y atractiva la organización de proyectos. Sin embargo, estudios existentes como (García Borgoñón, Barcelona, García García, Alba, y Escalona, 2014) indican que estas notaciones son complejas de entender y utilizar y aún están evolucionando, por lo que finalmente se ha descartado.

A continuación, vamos a desarrollar algunos de los procesos de la Tabla 1 y utilizaremos algunas propuestas sencillas del PMBOK para abordarlos. Es importante resaltar que los datos aportados en los siguientes apartados corresponderían a las decisiones tomadas por Rosa y el equipo organizador para la realización del Proyecto Barbacoa. 


\begin{tabular}{|c|c|}
\hline Procesos & Preguntas claves a las que tendrán que dar respuesta nuestro alumnado \\
\hline Desarrollarel Actade Constitución & $\begin{array}{l}\text { ¿Tenemos claro lo que queremos hacer? ¿Lo escribimos para informar al } \\
\text { resto? ¿Quién se encargará de todo? }\end{array}$ \\
\hline Identificar a los interesados & $\begin{array}{l}\text { ¿Quiénes asistirán? ¿Quiénes tienen mayor influencia? ¿Quién puede poner } \\
\text { problemas? ¿Qué podemos hacer para que todos estén contentos? }\end{array}$ \\
\hline Recopilar requisitos & ¿Qué queremos hacer exactamente? ¿Qué esperamos? \\
\hline Definir el alcance & ¿Qué cosas haremos y qué otras cosas no haremos? \\
\hline Controlar el alcance & $\begin{array}{l}\text { ¿Estamos haciendo todo lo que nos habíamos propuesto en el plan? ¿Falta } \\
\text { algo? ¿Estamos haciendo algo que no habíamos decidido? }\end{array}$ \\
\hline Definir las actividades & ¿Qué actividades vamos a realizar? ¿Quiénes serán los responsables? \\
\hline Realizar el control de la calidad & $\begin{array}{l}\text { ¿Las bebidas y la comida que hemos comprado están buenas? ¿Estamos } \\
\text { teniendo suficientes vasos y platos? }\end{array}$ \\
\hline Duración de las actividades & ¿Cuánto tiempo tenemos para cada actividad? \\
\hline Desarrollar el cronograma & $\begin{array}{l}\text { ¿Hacemos un esquema para ver cómo se llevarán a cabo las actividades en } \\
\text { el tiempo? }\end{array}$ \\
\hline Controlar el cronograma & $\begin{array}{l}\text { ¿Nos estamos pasando de tiempo respecto de lo que habíamos previsto? } \\
\text { ¿Cuál es el tiempo máximo que tenemos? }\end{array}$ \\
\hline Determinar el presupuesto & ¿Cuánto nos vamos a gastar y en qué? ¿En total, cuánto nos vamos a gastar? \\
\hline Controlar los costes & $\begin{array}{l}\text { ¿Estamos gastando más de lo previsto? ¿Tenemos algún remanente por si hay } \\
\text { gastos extras? }\end{array}$ \\
\hline Planificar las comunicaciones & $\begin{array}{l}\text { ¿Cómo vamos a estar conectados? ¿Usamos WhatsApp? ¿Abrimos una } \\
\text { página de Facebook? ¿Todos vamos a estar informado de todo? }\end{array}$ \\
\hline Identificar los riesgos & $\begin{array}{l}\text { ¿Qué puede salir mal? ¿Hará buen tiempo? ¿Faltará mucha gente? ¿Vendrán } \\
\text { más de los previstos? ¿Nos dejarán hacerla? ¿Conseguiremos el dinero? }\end{array}$ \\
\hline Planificar la calidad & $\begin{array}{l}\text { ¿Vamos a comprar muchas bebidas? ¿Y comidas? ¿Compraremos bebidas } \\
\text { y comidas de marcas blancas? ¿Pondremos algunos dulces y chucherías? } \\
\text { ¿Estaremos de pie o pondremos sillas? ¿Hay alguien con alguna intolerancia } \\
\text { a algo parecido? }\end{array}$ \\
\hline Cerrar el proyecto & $\begin{array}{l}\text { ¿Hemos hecho todo lo que habíamos pensado? ¿A qué hora quedamos } \\
\text { para recoger y cerrar las cuentas? ¿Ha habido algún extra con el que no } \\
\text { contábamos? ¿Estamos contentos? ¿Hacemos lista con lo que más nos ha } \\
\text { gustado y con los problemas que hemos tenido? }\end{array}$ \\
\hline Cerrar las adquisiciones & $\begin{array}{l}\text { ¿Hemos recibido todo lo que habíamos encargado? ¿Se ha pagado todo? ¿Hay } \\
\text { que devolver algo (envases, sillas, etc.)? }\end{array}$ \\
\hline
\end{tabular}

Tabla 1. Procesos y preguntas usuales a las que debemos dar respuesta para la realización del Proyecto Barbacoa. Fuente: (PMI, 2014) y elaboración propia 


\section{Subproceso Desarrollar el Acta de Constitución}

- ¿Tenemos claro lo que queremos hacer?

- ¿Lo escribimos para informar al resto?

- ¿Quién se encargará de todo?

Tabla 2. Desarrollo del acta de constitución

Este subproceso, Tabla 2, pertenece al Área de Conocimiento de la Gestión de la Integración del Proyecto y al Grupo de Procesos de Inicio

Anteriormente ya se ha indicado qué es un Acta de Constitución y el acta de nuestro Proyecto se parecería bastante a la descripción aportada por el alumnado ampliada con las respuestas a las preguntas planteadas en la Tabla 1. A continuación desarrollaremos algunos de los subprocesos contestando a dichas preguntas.

\begin{tabular}{|c|c|}
\hline \multirow{2}{*}{$\begin{array}{c}\text { Subproceso Identificar a los } \\
\text { interesados }\end{array}$} & $\cdot \quad$ ¿Quiénes asistirán? \\
& $\cdot \quad$ ¿Quiénes tienen mayor influencia? \\
& $\cdot \quad$ ¿Quiénes pueden poner problemas? \\
\hline
\end{tabular}

Tabla 3. Identificación de los interesados

Este subproceso, Tabla 3, pertenece al Área de Conocimiento de la Gestión de los Interesados del Proyecto y al Grupo de Procesos de Inicio (PMI, 2014).

Los interesados de un proyecto son personas o entidades que tienen algún interés en el proyecto, bien para que sea un éxito o bien para que sea un fracaso.

Puede parecer que todos los interesados del Proyecto Barbacoa son aquellos que quieren ir, pero también estarán los que quieran ir pero no se les permita (por ejemplo, alumnado de otros grupos). Estos también son interesados en el Proyecto y como tal tendremos que considéralos por si pudieran influir negativamente sobre el mismo.

En la Tabla 4, hemos recogido los interesados más importantes del Proyecto Barbacoa indicando para cada uno: el papel que juega, el impacto que tiene (alto, medio o bajo), es decir, en qué medida puede afectar al éxito o fracaso del proyecto, la prioridad o importancia que tiene (alta, media o baja), así como la estrategia de gestión a seguir. Entendemos por estrategia de gestión las acciones que llevaremos a cabo con los interesados para aumentar su apoyo al Proyecto y minimizar cualquier impacto negativo que pudieran tener sobre el mismo.

Para nuestro Proyecto los interesados más importantes además de Rosa y el equipo organizador son: los que tienen mucho interés por saber lo que haremos, aunque no pueden influir mucho (poco poder) como los vecinos próximos de José Manuel y alumnos de otros cursos. A los vecinos le 
propondremos un horario de finalización que sea razonable y procuraremos que nos conozcan para que vean que somos responsables. A los compañeros de otras clases le comentaremos cómo ha salida la barbacoa ya que tienen interés en hacer algo parecido. Es muy importante para poder hacer la barbacoa hablar con los padres de José Manuel ya que, aunque tienen poco interés en los que hagamos, sin su permiso no podríamos realizar la barbacoa.

\begin{tabular}{|c|c|c|c|c|}
\hline Interesado & Papel que juega & Impacto & Prioridad & Estrategia \\
\hline $\begin{array}{l}\text { Equipo } \\
\text { organizador }\end{array}$ & Realizar el plan definido & Alto & Alta & $\begin{array}{c}\text { Informar continuamente del trabajo } \\
\text { que se está realizando y de posibles } \\
\text { desviaciones en el plan }\end{array}$ \\
\hline Rosa & $\begin{array}{l}\text { Responsable del plan } \\
\text { definido }\end{array}$ & Alto & Alta & $\begin{array}{l}\text { Estar informada del trabajo realizado } \\
\text { por cada miembro del equipo y } \\
\text { aceptar los cambios a realizar }\end{array}$ \\
\hline $\begin{array}{l}\text { Resto del } \\
\text { alumnado de la } \\
\text { clase }\end{array}$ & Disfrutar de la barbacoa & Medio & Media & $\begin{array}{l}\text { Informar sobre el plan y los avances } \\
\text { producidos }\end{array}$ \\
\hline $\begin{array}{l}\text { Padres del } \\
\text { anfitrión }\end{array}$ & $\begin{array}{c}\text { Dejar la zona ajardinada } \\
\text { de la casa para realizar la } \\
\text { barbacoa }\end{array}$ & Alto & Alta & $\begin{array}{l}\text { Informar sobre las medidas que se } \\
\text { tomarán para que no se produzcan } \\
\text { conflictos ni destrozos }\end{array}$ \\
\hline $\begin{array}{l}\text { Padres de } \\
\text { los alumnos } \\
\text { asistentes }\end{array}$ & $\begin{array}{c}\text { Dar permiso para asistir a la } \\
\text { barbacoa }\end{array}$ & Medio & Baja & $\begin{array}{l}\text { Informar sobre el lugar y horarios de la } \\
\text { barbacoa }\end{array}$ \\
\hline $\begin{array}{l}\text { Vecinos del } \\
\text { anfitrión }\end{array}$ & $\begin{array}{l}\text { Vigilar de que no se } \\
\text { produzcan conflictos o } \\
\text { volumen excesivo de ruidos }\end{array}$ & Bajo & Baja & Informar de los horarios \\
\hline $\begin{array}{l}\text { Alumnado de } \\
\text { otros grupos }\end{array}$ & $\begin{array}{l}\text { Interés en conocer el } \\
\text { proyecto }\end{array}$ & Bajo & Baja & $\begin{array}{l}\text { Informar sobre la organización y } \\
\text { lecciones aprendidas }\end{array}$ \\
\hline
\end{tabular}

Tabla 4. Registro de Interesados del Proyecto Barbacoa. Fuente: (PMI, 2014) y elaboración propia

En la Figura 2, se muestra una Matriz Interés/Poder (PMI, 2014) para conocer la prioridad e impacto de cada uno de los interesados. Esta matriz nos permite clasificar cada uno de los interesados en base al interés y el poder (de menor a mayor para ambas variables) que pudiera tener sobre el Proyecto y nos permite analizar y visualizar rápidamente quiénes serán los interesados claves. Para el Proyecto Barbacoa, según la matriz, vemos que los padres del anfitrión pueden tener poco interés por la barbacoa, pero si, en algún momento, nos deniega el permiso para hacerlo en su jardín, o no se podría realizar el Proyecto o tendríamos que replantearlo de nuevo. Por otro lado, todo el equipo tiene un gran interés en que se realice el Proyecto y de ellos depende su éxito. 


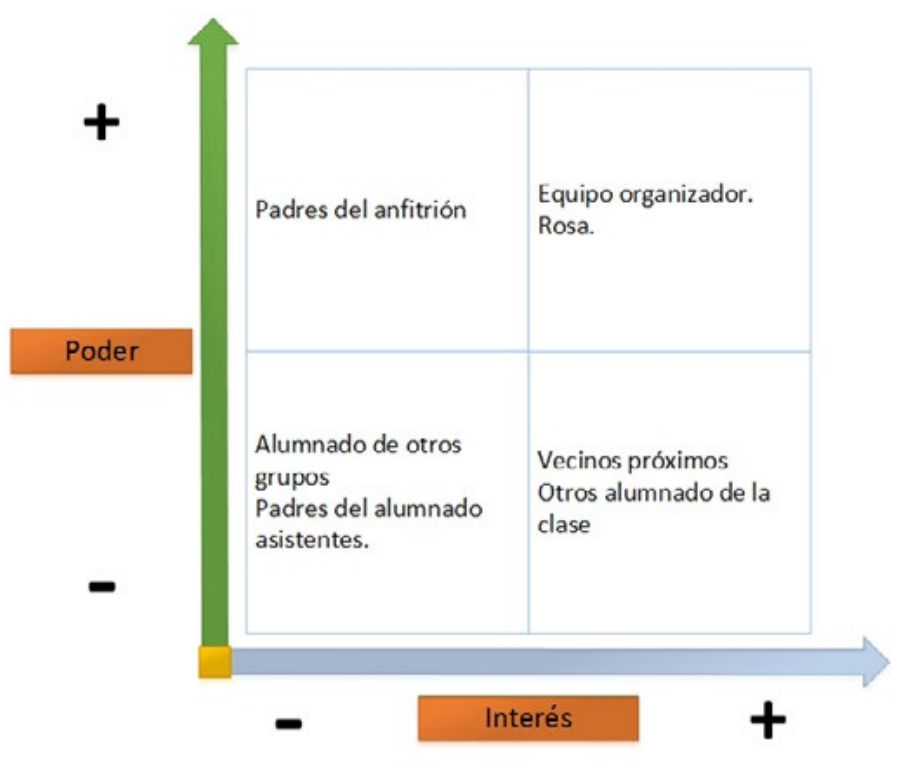

Figura 2. Matriz Poder/Interés para analizar la prioridad de los interesados en el Proyecto Barbacoa. Fuente: (PMI, 2014) y elaboración propia

Los criterios definidos, intervalos de valoración y decisiones tomadas en la Tabla 4 y Figura 2 se acuerdan entre el equipo organizador y la responsable del Proyecto.

\begin{tabular}{|c|c|}
\hline $\begin{array}{c}\text { Subproceso Determinar el } \\
\text { presupuesto }\end{array}$ & $\cdot \begin{array}{l}\text { ¿Cuánto nos vamos a gastar y en qué? } \\
\text { ¿En total, cuánto nos vamos a gastar? }\end{array}$ \\
\hline
\end{tabular}

Tabla 5. Determinación del presupuesto

Este subproceso, Tabla 5, pertenece al Área de Conocimiento de la Gestión del Coste Proyecto y al Grupo de Procesos de Planificación del PMBOK.

Para elaborar un presupuesto tendremos que analizar las partidas de las que constará (recursos humanos, consumibles, espacios, mantenimiento, costes de estructura, financieros y un largo etc.). Nos centraremos solo en las partidas necesarias para el Proyecto Barbacoa.

El alumnado ha hablado con amigos de otros grupos que también celebran fiestas parecidas y estiman que si lo hacen en casa de algún amigo que ya tiene barbacoa en el jardín se podrían gastar unos 400 euros. En la Tabla 6, se recoge el desglose del presupuesto.

\begin{tabular}{|c|c|}
\hline Concepto & Coste (€) \\
\hline $\begin{array}{c}\text { Bebidas (refrescos de varios tipos) } \\
\text { aceitunas, pan, etc.) }\end{array}$ & 120 \\
\hline $\begin{array}{c}\text { Otros (mantel de papel, servilletas, cubiertos, carbón, pastillas para encender la barbacoa, bolsas de fritas, palomitas, tortillas, } \\
\text { basura, etc.) }\end{array}$ & 30 \\
\hline Limpieza (si nosotros no lo hacemos) & 20 \\
\hline Otros para imprevistos & 50 \\
\hline Total & $\mathbf{4 0 0}$ \\
\hline
\end{tabular}


Como esperamos asistir todos, los 25 alumnos y alumnas de la clase, tendremos que aportar cada uno 16 euros.

Los padres de José nos dejarán que usemos su equipo de música para tener música ambiente en el jardín durante la barbacoa y las mesas y sillas que tienen por lo que no será necesario que llevemos nada de esto.

\begin{tabular}{|c|c|}
\hline \multirow{2}{*}{$\begin{array}{c}\text { Subproceso Identificar los } \\
\text { riesgos }\end{array}$} & ¿Qué puede salir mal? \\
& $\cdot \quad$ ¿Hará buen tiempo? \\
& $\cdot \quad$ ¿Faltará mucha gente? \\
& $\cdot \quad$ ¿Nondrán más de los previstos? \\
& $\quad$ ¿Conseguiremos dinero? \\
\hline
\end{tabular}

Tabla 7. Presupuesto del Proyecto Barbacoa. Fuente: elaboración propia

Este subproceso, Tabla 7, pertenece al Área de Conocimiento de la Gestión de los Riesgos del Proyecto y al Grupo de Procesos de Planificación del PMBOK.

Como cualquier proyecto que desarrollemos, siempre existe la posibilidad de que algo no salga como habíamos previsto.

Un aspecto que suele sorprender al alumnado es que hay riesgos positivos además de los riesgos negativos. Por ejemplo, para proyectos de este tipo un riesgo positivo sería la asistencia de más personas de las esperadas al evento (jesto no ocurrirá en el Proyecto Barbacoa porque han puesto el requisito de que solo asistirá el alumnado de la clase!). Esto implicaría que nuestro poder de convocatoria ha sido grande y como tal hay que verlo como algo positivo. Pero este riesgo, si no se gestiona adecuadamente, puede hacer que se consuman los recursos antes de lo previsto o que no se tenga espacio suficiente para estar con comodidad.

Para cada uno de los posibles riesgos que identifiquemos en un proyecto tenemos que valorar el impacto que puede tener sobre el proyecto, la probabilidad de que se produzca y la estrategia de gestión a seguir, es decir, cómo tenemos que actuar para que el riesgo no se produzca o bien minimicemos su impacto en el caso de que se produzca.

En la Tabla 8, se muestran los posibles riesgos del Proyecto Barbacoa. Para cada uno de los riesgos definidos se valora el posible Impacto en el Proyecto, valorándolo como Alto si dicho riesgo puede poner en peligro la realización de la barbacoa, Medio para un riesgo que permite realizar la barbacoa, pero con cambios importantes y Bajo para riesgos que pueden ser solventados fácilmente en caso de producirse. La probabilidad, en la Tabla 8, describe la posibilidad que tiene el riesgo de ocurrir o no valorándola como Probable (si la posibilidad de ocurrencia es mayor del 50\%) o Improbable (si la posibilidad de ocurrencia en menor del 50\%) y la estrategia de gestión propuesta. 


\begin{tabular}{|c|c|c|c|}
\hline Riesgo & Impacto & Probabilidad & Estrategia (¿Qué haremos?) \\
\hline Riesgo de lluvia & Alto & Improbable & $\begin{array}{l}\text { Solicitar permiso para trasladar la barbacoa al garaje, que } \\
\text { está cubierto }\end{array}$ \\
\hline Sobran víveres & Bajo & Probable & $\begin{array}{l}\text { Al final de la barbacoa se repartirán las sobras entre los } \\
\text { miembros del equipo }\end{array}$ \\
\hline Faltan víveres & Bajo & Improbable & $\begin{array}{c}\text { Tener anotado dónde está el súper más próximo para } \\
\text { comprar más víveres }\end{array}$ \\
\hline Falta de presupuesto & Medio & Improbable & $\begin{array}{l}\text { Ampliar la aportación de los asistentes con la cuota que } \\
\text { decida el equipo y recaudar lo más rápido posible }\end{array}$ \\
\hline $\begin{array}{l}\text { Asistentes que no han } \\
\text { pagado al comienzo de } \\
\text { la barbacoa }\end{array}$ & Bajo & Probable & $\begin{array}{c}\text { Enviarles mensajes antes de la barbacoa para recordárselo. } \\
\text { Si el motivo está justificado asumirlo con el presupuesto } \\
\text { que tengamos }\end{array}$ \\
\hline $\begin{array}{l}\text { Falta de trasporte para } \\
\text { todos }\end{array}$ & Medio & Improbable & $\begin{array}{c}\text { Hacer una lista de vehículos y de compañeros que no } \\
\text { tengan medios para ir y hacer una distribución. Si no, hablar } \\
\text { con algunos padres para que los lleven y recojan, ya que el } \\
\text { transporte público no nos vale }\end{array}$ \\
\hline $\begin{array}{l}\text { Asistencia de personas } \\
\text { conflictivas }\end{array}$ & Alto & Probable & $\begin{array}{c}\text { Designar a miembros del equipo para que estén pendientes } \\
\text { y procurar evitar encuentros entre ellas. Dejar claro que ante } \\
\text { cualquier conflicto entre los asistentes se les expulsará de } \\
\text { la barbacoa }\end{array}$ \\
\hline $\begin{array}{l}\text { No recoger los residuos } \\
\text { y demás elementos } \\
\text { utilizados al finalizar la } \\
\text { barbacoa }\end{array}$ & Medio & Probable & $\begin{array}{l}\text { Se dejará una cantidad acordada del presupuesto para } \\
\text { contratar a una persona que deje el jardín limpio. José } \\
\text { Manuel nos confirmará de si se ha hecho bien o no }\end{array}$ \\
\hline
\end{tabular}

Tabla 8. Registro de riesgos del Proyecto Barbacoa. Fuente: (PMI, 2014) y elaboración propia

\begin{tabular}{|c|c|}
\hline Subproceso Definir las actividades & $\begin{array}{l}\text { ¿Qué actividades vamos a realizar? } \\
\text { ¿Quiénes serán los responsables? }\end{array}$ \\
\hline $\begin{array}{c}\text { Subproceso Duración de las } \\
\text { actividades }\end{array}$ & $\cdot \quad$ ¿Cuánto tiempo tenemos para cada actividad? \\
\hline
\end{tabular}

Tabla 9. Definición de actividades

Estos subprocesos, Tabla 9, pertenecen al Área de Conocimiento de la Gestión del Tiempo del Proyecto y al Grupo de Procesos de Planificación del PMBOK.

Para la realización del cronograma de un proyecto es necesario, una vez definidos los requisitos y el alcance del mismo, en el que tendremos que indicar con claridad qué forma parte del proyecto y qué no lo forma, tenemos que realizar los siguientes subprocesos: Definir las actividades, Secuenciar las actividades, Estimar los recursos y la Duración de cada actividad y desarrollar el Cronograma. 
El alumnado seguramente realizará, al menos, los subprocesos de Definir las actividades y Estimar su duración. En la Tabla 10, hemos recogido las principales actividades que tienen que realizar indicando para cada una las restricciones de tiempo que tienen y quién será la persona responsable. El Proyecto comenzará el día 5 de junio y finalizará el día 25 del mismo mes. Rosa controlará que todas las actividades se realicen según lo previsto

\begin{tabular}{|c|c|c|}
\hline Tareas & Tiempo (días junio) & Responsable \\
\hline $\begin{array}{c}\text { Reunión inicial para concretar los } \\
\text { detalles y asignar trabajo }\end{array}$ & El día 5 a las $17 \mathrm{~h}$ & Rosa. Asistirá todo el equipo \\
\hline Recaudar el dinero & Desde el 6 al día 23 & Mario Ramírez \\
\hline Comprar la comida & Entre los días 20 y 23 & Juana Muñiz \\
\hline Comprar la bebida & Entre los días 20 y 23 & Alejandro Silva \\
\hline Comprar el resto de cosas & Entre los días 20 y 23 & Alejandro Silva \\
\hline Reunión de seguimiento & Día 19 a las $17 \mathrm{~h}$ & Rosa. Asistirá todo el equipo \\
\hline Elaborar carteles informativos & Desde el día 6 al 17 & Juana Muñiz \\
\hline Controlar el transporte & Desde el 6 al día 23 & Nuria Fernández \\
\hline Controlar las comunicaciones & Desde el 6 al día 25 & Nuria Fernández \\
\hline $\begin{array}{l}\text { Preparar y controlar la parrilla durante } \\
\text { la barbacoa }\end{array}$ & Día 24 & José Manuel Pérez \\
\hline Disfrutar de la barbacoa & Día 24 entre las 14 h y 01 h del día 25 & Toda la clase \\
\hline $\begin{array}{c}\text { Repartir la comida y bebida durante la } \\
\text { barbacoa }\end{array}$ & Día 24 entre las 14 h y 01 h del día 25 & $\begin{array}{c}\text { Todo el equipo salvo José Manuel } \\
\text { Pérez }\end{array}$ \\
\hline Recoger y limpiar el jardín & Día 25 a las $12 \mathrm{~h}$ & Equipo completo \\
\hline Recoger Lecciones Aprendidas & Día 25 una vez finalizada la limpieza & Rosa y todo el equipo \\
\hline
\end{tabular}

Tabla 10. Relación de actividades, restricciones temporales y responsable. Fuente: elaboración propia

Subproceso Planificar las comunicaciones
- ¿Cómo vamos a estar conectados?

- ¿Usamos WhatsApp?

- ¿Abrimos una página de Facebook?

- ¿Todos vamos a estar informado de todo?

Tabla 11. Planificación de comunicaciones

Este proceso, Tabla 11, pertenece al Área de Conocimiento de la Gestión de las Comunicaciones del Proyecto y al Grupo de Procesos de Planificación del PMBOK.

Como se ha visto en la identificación de los interesados en el Proyecto, no todos tienen las mismas 
implicaciones, por tanto, las necesidades de comunicación entre ellos también serán diferentes. Nuestro alumnado tendrá que decidir: qué información va a recibir el equipo organizador, los asistentes a la barbacoa y el resto de interesados; y cómo se va hacer, es decir, qué herramienta van a utilizar para cada grupo. Rosa y el equipo tendrán que decidir si solamente utilizarán grupos de WhatsApp, si utilizarán alguna herramienta para definir un cronograma y hacer el presupuesto, si montarán o no una página Facebook abierta a todos o si utilizarán alguna herramienta para almacenar información sobre el trabajo que se está desarrollando.

Con el uso intensivo que se hace hoy día de los móviles y tablets seguro que nuestros jóvenes utilizarán algunas herramientas para cubrir sus necesidades de comunicación. En la sección siguiente comentamos alguna de ellas.

\section{Propuesta de algunas herramientas y lecturas recomendadas}

A continuación, se presentarán algunas herramientas de soporte al Proyecto Barbacoa y algunas lecturas que permitirán al alumnado y al profesorado interesado entusiasmarse con la gestión y dirección de proyectos.

\subsection{Herramientas de soporte al Proyecto Barbacoa}

¿Utilizará el alumnado sus móviles o tablets para organizar la barbacoa? Seguro que sí. Por ello, identificaremos algunas herramientas de uso común que permiten dar soporte a las preguntas y respuestas, planteadas en el Apartado 2 para organizar el Proyecto, y son accesibles para todos los públicos.

Proponemos, por tener funcionalidades diferentes y por su facilidad de aplicación, WhatsApp (https:// web.whatsapp.com), Trello (https://trello.com) y Google Drive(https://drive.google.com). Lógicamente, se puede emplear cualquiera otra, por ejemplo, Facebook en lugar de WhatsApp para centralizar la comunicación del equipo. Estas herramientas cuentan con interfaz web y aplicaciones específicas para móviles y tablets, con lo cual se integran fácilmente en los hábitos digitales del alumnado. Los recursos disponibles, de manera gratuita, de estas herramientas son más que suficientes para gestionar el Proyecto, aunque Trello y Google Drive también ofrecen alternativas de pago.

Estas herramientas también permiten irradiar información y minimizar la posibilidad de que surjan silos de información en el proyecto. Un silo de información se genera cuando una persona o grupo de personas aísla información necesaria para que otros grupos de personas actúen (Figura 3). Por ejemplo, un silo de información podría surgir si los encargados de hacer la compra no indican cuánto han comprado, por lo que otros miembros del equipo organizador no podrían saber si se han realizado las compras previstas y cuánto se lleva gastado. 

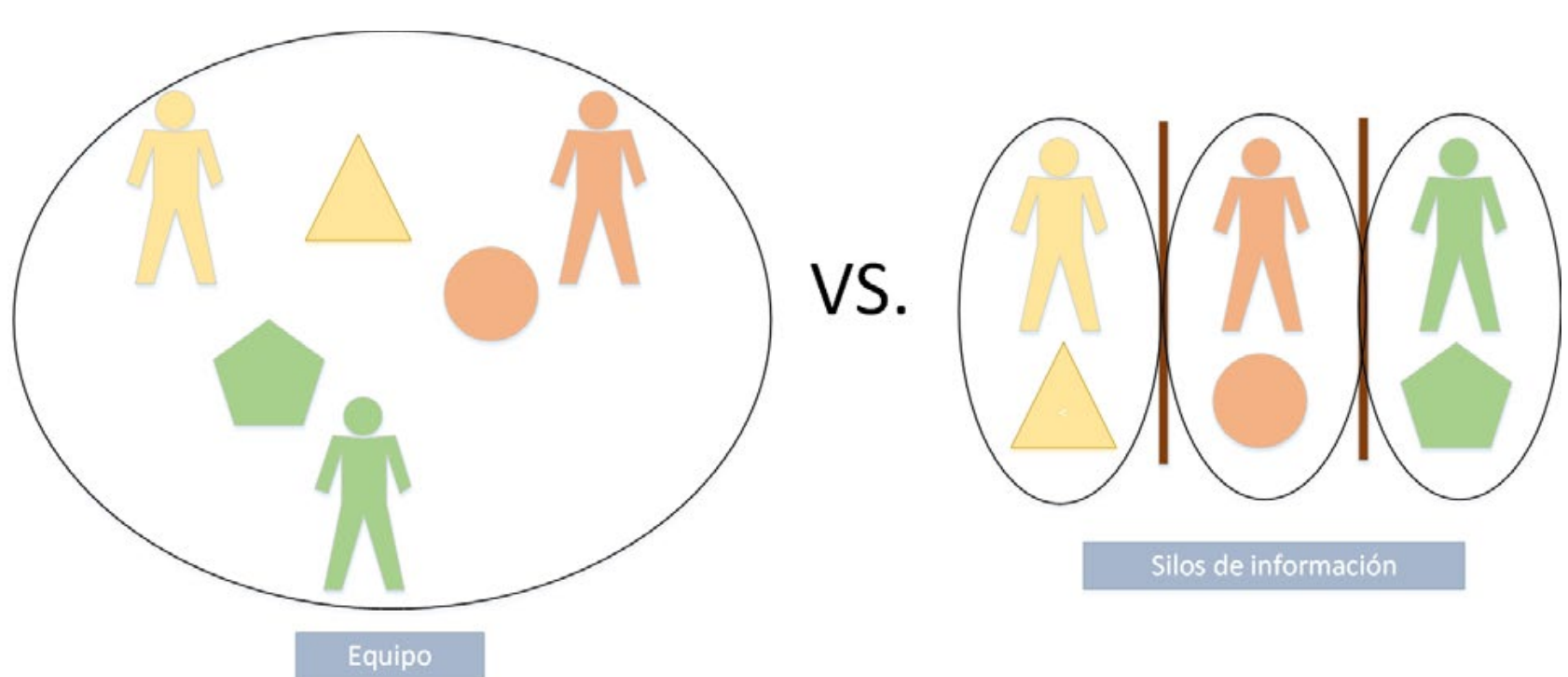

Figura 3. Organización que comparte información (equipo) vs. silos. Fuente: elaboración propia

WhatsApp es una de las herramientas más utilizadas por los jóvenes (y menos jóvenes) para comunicarse, por lo que es una de las mejores candidatas en este segmento. WhatsApp permite organizar grupos de tal manera que los mensajes que se escriban o los archivos que se compartan estén solo disponibles para los miembros de dichos grupos.

La segunda herramienta propuesta es Trello. Esta es una herramienta web, que cuenta también con una aplicación específica para dispositivos móviles, que permite trabajar con tableros y tarjetas. Utilizando estos dos sencillos elementos, Trello permite organizar las tareas de un equipo, visualizar su avance y compartir esta información en un grupo de trabajo.

En Trello definimos listas de tareas que se van colocando de izquierda a derecha. Todas las tareas estarán dentro de alguna lista de tareas (por ejemplo: tareas pendientes, tareas en ejecución o tareas terminadas) e irán viajando de izquierda a derecha a través de las listas a medida que se vayan completando. Además, en cada tarea se puede indicar: la fecha límite para su realización, los responsables y participantes, colores para agruparlas en tipos de tarea o prioridades, e iconos. En la Figura 4 hemos recogido un ejemplo con un subgrupo de actividades. Hemos asignado el amarillo para las actividades relacionadas con temas económicos, naranja para las relacionadas con las compras y morado para las relacionadas con el transporte de los asistentes. Inicialmente todas las actividades definidas estarían a la izquierda. En la Figura 4 (web) y Figura 5 (tablet) podemos ver el avance del trabajo en un momento dado. Se puede comprobar que, a la vista de este tablero, es posible conocer el estado del Proyecto sin necesidad de preguntar a los miembros del equipo. Una vez que el Proyecto haya finalizado todas las tareas estarán en la columna de la derecha. 


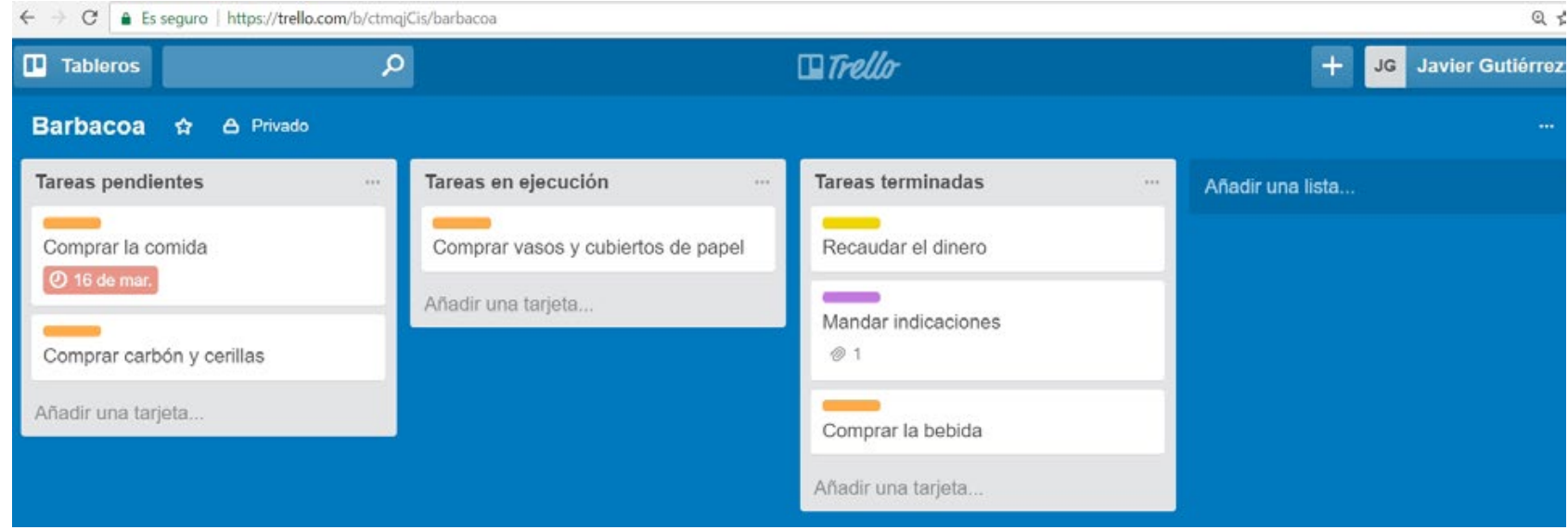

Figura 4. Interfaz web del tablero de Trello para gestionar las actividades del Proyecto Barbacoa. Fuente: Elaboración propia

Trello evita los silos de información permitiendo que cualquier persona interesada pueda ver, pero no modificar si no tiene los permisos necesarios, los tableros. Además, ofrece la posibilidad de invitar a otros usuarios para que todos, en este caso el equipo, puedan trabajar con el mismo tablero.

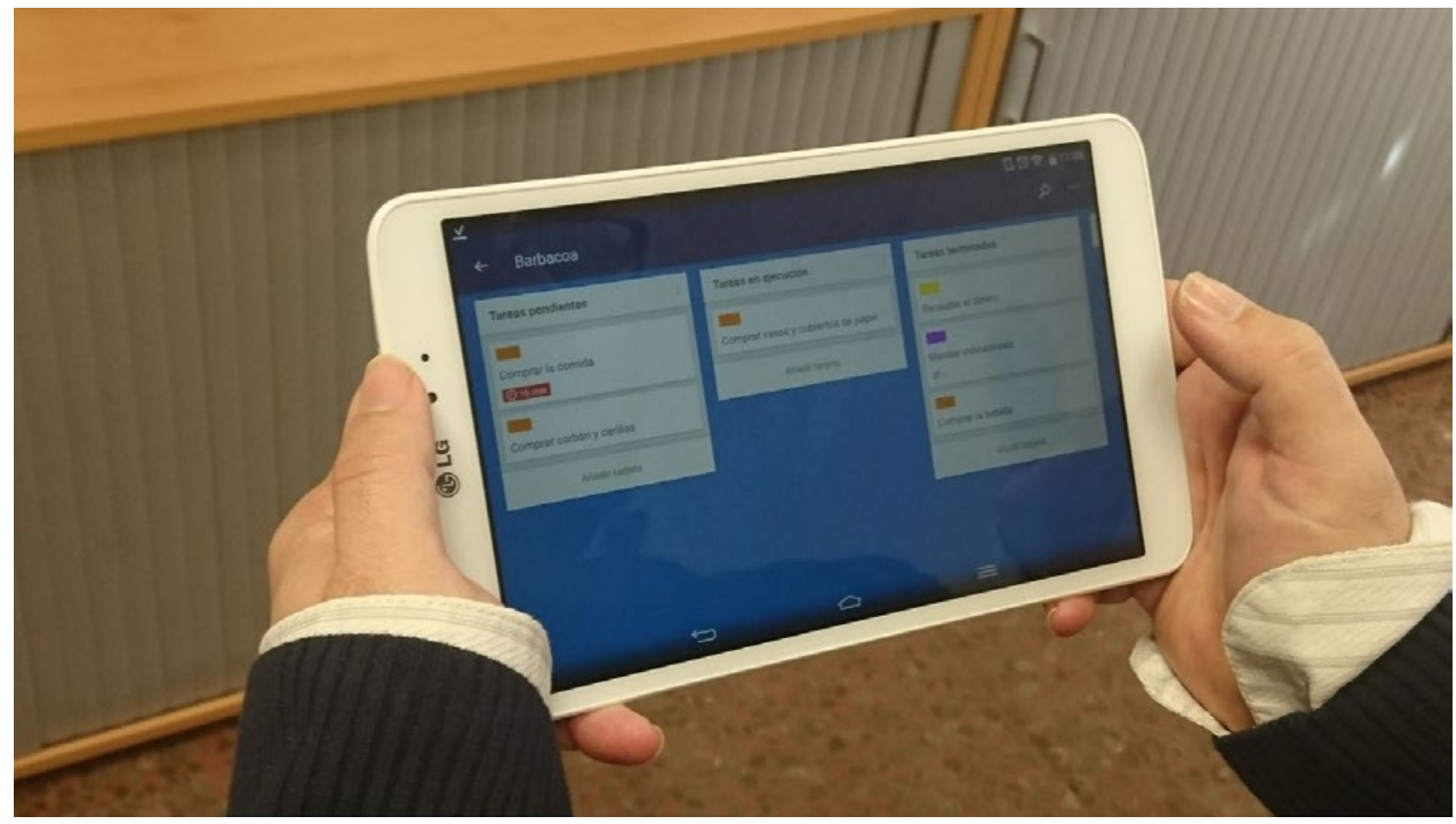

Figura 5. Utilización del tablero de Trello mediante Tablet para gestionar las actividades del Proyecto Barbacoa. Fuente: Elaboración propia

Con las dos herramientas anteriores ya es posible organizar la comunicación del Proyecto y llevar toda la gestión de tareas. Sin embargo, aún quedan algunos detalles que son difíciles de gestionar, por ejemplo: ¿cuántos coches tenemos?, ¿cuál es la lista de conductores?, etc. Esta información, o cualquier otra, podría estar disponibles para todos los interesados del Proyecto, incluso, se podría elaborar de manera colaborativa. Por ello proponemos una tercera herramienta, Google Drive, que puede utilizarse para lograr este objetivo.

Google ofrece como parte de sus herramientas en línea la herramienta Google Drive, que permite 
elaborar documentos de texto u hojas de cálculo a través de un navegador y de manera colaborativa. El documento puede enlazarse con una tarea de Trello, de manera que se pueda abrir y editar desde la propia tarea. Además del enlace a través de Trello, cualquier persona que reciba un enlace podría acceder y/o editar un documento de Google Drive. Google también ofrece una aplicación específica de Google Drive para dispositivos móviles.

\subsection{Lecturas recomendadas}

En el ámbito de la difusión de la gestión y dirección de proyectos para estudiantes existen diferentes propuestas. En este apartado se presentan algunas de ellas.

La novela (Kim, Behr y Spafford, 2014) está basada en hechos reales en la que se muestra la vida de Bill, recién ascendido a responsable de desarrollo de sistemas de información en una gran empresa dedicada a la fabricación de accesorios para coches. Desde el momento en que todo se desborda en su trabajo, por culpa de una mala gestión de proyectos, Bill comienza a analizar cuáles son las peores y mejoras prácticas llevadas a cabo. El libro está muy enfocado a proyectos de sistemas de información y pone de relieve, de manera novelada, las ventajas de Lean y Devops (Kim, Willis, Debois y Humble, 2016).

El libro (Graham, 2015) pertenece a la famosa serie de libros para "Dummies". Un Dummy es un muñeco que representa a un humano y en algunos contextos esta palabra significa principiante o alguien de pocas luces. Este libro expone el trabajo que compete a un director de proyectos y da recetas desde una perspectiva no técnica para intentar conseguir el éxito, es decir, según el libro, finalizar los proyectos en el tiempo y presupuesto previsto. El libro cuenta ya con cuatro reediciones e incluso una edición adaptada al Reino Unido, lo cual es un indicio del interés de la gestión de proyectos desde una perspectiva sencilla y práctica.

El artículo (Liegel, 2007) presenta una serie de plantillas para facilitar la recogida de información de nuestro proyecto. Por ejemplo, se proponen plantillas para recoger los objetivos del proyecto, para identificar a los interesados, definir el plan de comunicaciones, etc. No se incluyen ejemplos ni descripciones en detalle de esos elementos.

La serie de cuatro libros (y de un quinto próximamente) Project Kids Adventures (Nelson, 2013) también describe la gestión y dirección de proyectos para un público muy joven. Existe un proyecto de traducción de estos libros al castellano.

El libro (Gómez, 2016) presenta varios aspectos de gestión de proyectos relacionándolos con los personajes y sucesos de los libros y serie de Juego de Tronos. Es una lectura muy recomendable, pero antes habría que ver la serie o leerse el libro de Juego de Tronos. 


\section{Conclusiones}

En este trabajo se ha querido mostrar el conocimiento natural que tiene el alumnado sub 18 para la dirección y gestión de proyectos sin que seguramente ni ellos mismos lo sepan; y el potencial que puede tener este conocimiento previo para definir sus futuros planes formativos y profesionales. Para ello, se ha propuesto organizar una barbacoa utilizando como base las directrices y buenas prácticas recogidas en el estándar internacional Guía de los Fundamentos para la Dirección de Proyectos, más conocido como PMBOK, desarrollado por el PMI. Hemos simultaneado conceptos básicos sobre gestión y dirección de proyectos con lo que haría el alumnado para organizar el Proyecto Barbacoa. Como soporte a este proyecto y conociendo la utilización que hacen los jóvenes de sus móviles y tablets, se han mostrado algunas herramientas gratuitas y sencillas para facilitar la realización del plan del proyecto, así como su control y ejecución. Hemos considerado de interés presentar algunas lecturas amenas dirigidas al alumnado, pero también para aprender, nosotros mismos como docentes, y motivarlos.

En definitiva, con este trabajo queremos plantear la siguiente reflexión: ¿por qué esperar a los últimos cursos de la carrera universitaria o módulos superiores para enseñarle a nuestro alumnado buenas prácticas sobre este ámbito de conocimiento? Proponemos empezar su enseñanza desde el inicio de su formación para facilitarles la planificación y ejecución de sus primeras actividades formativas y mostrar la importancia que tiene para los jóvenes (iy para los no tan jóvenes!) aplicar estas buenas prácticas en la toma de decisiones, tanto en nuestras actividades cotidianas como en las profesionales.

\section{Agradecimientos}

Este trabajo ha sido apoyado por el proyecto POLOLAS (TIN2016-76956-C3-2-R) y por la Red SoftPLM (TIN2015-71938-REDT) del Ministerio de Economía y Competitividad.

\section{Referencias}

García Borgoñón, L., Barcelona, M. A., García García, J. A., Alba, M., \& Escalona, M. J. (2014). Software process modelling languages: A systematic literature review. Information and Software Technology, 56(2), 103-116. doi: https://doi.org/10.1016/j.infsof.2013.10.001

García-Peñalvo, F. J. (2016). Proyecto TACCLE3 - Coding. En F. J. García-Peñalvo \& J. A. Mendes (Eds.), XVIII Simposio Internacional de Informática Educativa, SIIE 2016 (pp. 187-189). Salamanca, España: Ediciones Universidad de Salamanca. 
Gómez, J. (2016). El Juego de Tronos de los Proyectos: 15 Lecciones magistrales sobre Liderazgo y Dirección de Proyectos exitosa. USA: Createspace Independent Publishing Platform.

Graham, N. (2015). Project Management for Dummies. USA: John Wiley \& Sons Inc.

Kim, G., Behr, K., \& Spafford, G. (2014). The Phoenix Project: A Novel About IT, DevOps, and Helping Your Business Win. USA: It Revolution Press.

Kim, G., Willis, J., Debois, P., \& Humble J. (2016). The Devops Handbook: How to Create World-Class Agility, Reliability, and Security in Technology Organizations. USA: It Revolution Press

Liegel, K. M. (2007). Empowering Kids through Project Skills. Recuperado el 04 de marzo de 2017 de https://www.pmi.org/learning/library/empowering-kids-through-project-skills-7220

Nelson, G. M. (2013) The Ultimate Tree House Project: Volume 1 (Project Kids Adventures). USA: Createspace Independent Publishing Platform.

Project Management Institute (PMI) (2014) Guía de los fundamentos para la Dirección de Proyectos (Guía del PMBOK®). USA: Project Management Institute, Inc. 